\title{
A COMPARATIVE EVALUATION OF PETROFF'S METHOD AND NALC-NaOH DIGESTION DECONTAMINATION PROCEDURE FOR THE ISOLATION OF MYCOBACTERIA FROM CLINICAL SPECIMENS IN A TERTIARY CARE HOSPITAL
}

\author{
Kawal Preet Kaur', Harender Simar², Pardaman Singh Setia ${ }^{3}$, Bharti Arora ${ }^{4}$, Garima Mehta ${ }^{5}$, Priyanka Meel ${ }^{6}$ \\ ${ }^{1}$ Associate Professor, Department of Microbiology, Maharaja Agrasen Medical College, Agroha, Hisar. \\ ${ }^{2}$ Assistant Professor, Department of Microbiology, Maharaja Agrasen Medical College, Agroha, Hisar. \\ ${ }^{3}$ Assistant Professor, Department of Radiodiagnosis, Maharaja Agrasen Medical College, Agroha, Hisar. \\ ${ }^{4}$ Professor and HOD, Department of Microbiology, Maharaja Agrasen Medical College, Agroha, Hisar. \\ ${ }^{5}$ Demonstrator, Department of Microbiology, Maharaja Agrasen Medical College, Agroha, Hisar. \\ ${ }^{6}$ Demonstrator, Department of Microbiology, Maharaja Agrasen Medical College, Agroha, Hisar.
}

ABSTRACT
BACKGROUND
Tuberculosis is the leading cause of death from a curable infectious disease.[1] Examination by mycobacterial culture provides the
only definitive diagnosis of tuberculosis. ${ }^{[3]}$ Isolation of Mycobacteria from specimens contaminated with normal flora like sputum
poses a problem in view of high nutritive value of the egg media used for mycobacterial culture. Different laboratories use different
decontamination techniques. No single technique is entirely satisfactory. Hence, this study was conducted to compare the Petroff's
method and NALC-NAOH (N-Acetyl-L-cysteine $\mathrm{NaOH}$ ) method digestion decontamination procedures.

\section{MATERIALS AND METHODS}

This prospective study was conducted over a period of one year (1st August 2009 to 31st July 2010) in the Department of Microbiology, Dayanand Medical College and Hospital, Ludhiana. Samples received from various wards and intensive care units (ICUs) were processed for mycobacterial culture on 2 sets of Lowenstein-Jensen media using two decontamination proceduresPetroff's method and NALC-NaOH method. ${ }^{[4]}$ The isolation rate and contamination rate of growth on both the sets of LowensteinJensen media was compared.

\section{RESULTS}

The contamination rate of LJ media with Petroff's method was $20.31 \%$, while the contamination rate with NALC-NaOH method was $14.06 \%$. In our study, the rate of isolation was similar in both the decontamination procedures. The rate of isolation was $10.93 \%$ on both the sets of LJ media.

\section{CONCLUSION}

We observed that there was no significant difference in the isolation rate of mycobacteria with the use of either Petroff's method or NALC-NaOH method, while the contamination rate was higher with Petroff's method.

\section{KEYWORDS}

NALC-NaOH, TB, LJ Media, Petroff's Method.

HOW TO CITE THIS ARTICLE: Kaur KP, Simar H, Setia PS, et al. A comparative evaluation of Petroff's method and NALC-NaOH digestion decontamination procedure for the isolation of mycobacteria from clinical specimens in a tertiary care hospital. J. Evolution Med. Dent. Sci. 2017;6(69):4917-4921, DOI: 10.14260/Jemds/2017/1066

\section{BACKGROUND \\ Tuberculosis is the leading cause of death from a curable infectious disease. TB has affected mankind for over 5000 years and it still continues to be a leading cause of morbidity and mortality. The bacilli were discovered more than a century back by Sir Robert Koch in 1882 and effective drugs for treatment were available for more than half a century, globally more than 1.3 million people die of the disease every year. Nearly, one-third of the world's population is infected with TB bacilli, approximately $10 \%$ of them have a lifetime risk of developing TB disease.[1]}

Financial or Other, Competing Interest: None.

Submission 21-07-2017, Peer Review 14-08-2017,

Acceptance 21-08-2017, Published 28-08-2017.

Corresponding Author:

Dr. Harender Simar,

Assistant Professor,

Department of Microbiology,

Maharaja Agrasen Medical College, Agroha, Hisar.

E-mail:drsimar@gmail.com

DOI: $10.14260 /$ jemds $/ 2017 / 1066$

\section{(c) $($ ) $९$}

The emergence of resistance to drugs used to treat tuberculosis and particularly MDR-TB (Multi-Drug Resistant Tuberculosis) has become a significant public health problem in a number of countries and an obstacle to effective TB control.[2]

Examination by mycobacterial culture provides the only definitive diagnosis of tuberculosis. However, the usual microbiological techniques of plating clinical material on selective or differential culture media and subculturing to obtain pure cultures cannot be applied to tuberculosis bacteriology. Compared with other bacteria which typically reproduce within minutes, $M$. tuberculosis proliferate extremely slowly (generation time 18 - 24 hours). Furthermore, growth requirements are such that it will not grow on primary isolation on simple chemically defined media.[3]

Isolation of Mycobacteria from specimens contaminated with normal flora like sputum, poses a problem in view of high nutritive value of the egg media used for mycobacterial culture. Specimens are treated to kill various non-acid fast organisms by decontamination techniques. Different 
laboratories use different decontamination techniques. No single technique is entirely satisfactory. Hence, this study was conducted to compare the Petroff's method and NALC-NAOH (N-Acetyl-L-cysteine $\mathrm{NaOH}$ ) method digestion decontamination procedures.

\section{Aims and Objectives}

\section{This Study was designed}

1. To compare the contamination rates of two decontamination procedures, Petroff's Method and NALC$\mathrm{NaOH}$ method.

2. To compare the isolation rate of Mycobacteria on Lowenstein-Jensen's medium (LJ) using the above decontamination methods.

\section{MATERIALS AND METHODS}

This prospective study was conducted over a period of one year (1st August 2009 to 31st July 2010) in the Department of Microbiology, Dayanand Medical College and Hospital, Ludhiana. Samples received from various wards and intensive care units (ICUs) were processed for mycobacterial culture on 2 sets of Lowenstein-Jensen media using two decontamination procedures- Petroff's Method and NALC$\mathrm{NaOH}$ method.[4]

\section{Collection of Specimen/Pulmonary Specimens}

Sputum: Sputum was collected early in the morning before the patient had eaten or taken medication; 3 - $5 \mathrm{~mL}$ of sputum sample was collected in a clean, leak proof, disposable, widemouthed container.

\section{Endotracheal (ET) Secretions}

The ET secretion was collected using a 22-inch Ramson's 12 F suction catheter with a mucus extractor, which was gently introduced through the endotracheal tube for a distance of approximately $25-26 \mathrm{~cm}$. Gentle aspiration was then performed without instilling saline and the catheter was withdrawn from the endotracheal tube. After the catheter was withdrawn, $2 \mathrm{~mL}$ of sterile $0.9 \%$ normal saline was injected into it with a sterile syringe to flush the exudates into a sterile container for collection.

Bronchoalveolar Lavage (BAL): It required careful wedging of the tip of bronchoscope into an airway lumen isolating that airway from the rest of the central airway. Infusion of at least $120 \mathrm{~mL}$ of physiological saline in several (3 to 6) aliquots was required for adequate sampling of pulmonary segment. The aspirate was then collected in a sterile, robust and leak proof container.

\section{Extrapulmonary Specimens}

Body fluids like cerebrospinal fluid (CSF), pleural fluid, synovial fluid and ascitic fluid were collected taking all aseptic precautions in a sterile container.

\section{Pus}

Pus and discharge from wounds were collected in sterile container using cotton tipped swabs/ syringes. Tissue specimens were collected without preservatives or fixatives.

\section{Transportation}

The samples were transported immediately to the microbiology laboratory for further processing.

\section{Direct Examination}

The smear was prepared on a new, clean, unscratched slide numbered with a diamond-point stylus. With the help of a wooden stick, the purulent portion was picked up and spread evenly to cover $2 / 3$ of the central portion of the slide using a continuous rotational movement. The slide was then placed for air drying. The smear was heat-fixed by flaming. The smears were prepared directly from the sample and after concentration procedures and subjected to Ziehl-Neelsen (ZN) staining. The smears stained by ZN method were examined under oil immersion of light microscope.

\section{Smear Preparation after Concentration}

After digestion/decontamination, concentration and resuspension, the pellet was mixed well with a pipette and about 2 - 3 loopfulls or one drop was placed on a clean slide. The smear was spread to about $1 \frac{1}{2} \mathrm{~cm} \mathrm{x} 1 \mathrm{~cm}$ and allowed to air dry completely. The smear was heat-fixed by passing over the flame three to four times taking care not to overheat. The smear was then stained by the ZN method and examined under oil immersion of light microscope.

\section{Smears were Graded according to RNTCP Guidelines ${ }^{[5]}$}

A total of 64 samples were processed. The specimen processing was done using standard protocols.[6] The samples were processed in the microbiology laboratory within 2 hours of receipt. All the processing was done in a biosafety cabinet. Ziehl-Neelsen staining was done following standard procedures.[7]

0.1 to $0.25 \mathrm{~mL}$ of processed specimen was inoculated on L-J media and was incubated for 8 weeks. All culture bottles were examined daily for the first 7 days to detect rapid growers and to check for bacterial contamination. After that the cultures were examined twice weekly till 8 weeks. Preliminary identification of mycobacteria was done on the basis of rate of growth, colony characteristics, ZN staining and standard biochemical reactions.

The isolation rate and contamination rate of growth on both the sets of Lowenstein-Jensen media was compared. Sensitivity and Specificity of both smear and culture were also calculated

\section{Ethics}

No ethical issues are involved in this study, as there is no active intervention and no change in treatment protocol.

\section{Statistical Analysis}

The data obtained was analysed by computing tables of each variable and percentages were calculated. Pearson's chisquare test was applied. Sensitivity and Specificity of both smear and culture were also calculated.

\section{RESULTS}

From 64 suspected cases of tuberculosis admitted in various wards and ICUs, pulmonary and extra-pulmonary samples were received in the Department of Microbiology, DMCH. All the samples were subjected to staining by Ziehl-Neelsen and inoculated on 2 sets of Lowenstein-Jensen media after using two decontamination procedures- Petroff's Method and NALC-NaOH method.

The samples included sputum (36), Bronchoalveolar lavage (06), pleural fluids (07), urine (10), endometrial biopsy/ curettage (02), bone marrow biopsy (02), endotracheal secretions (01) and nasal secretions (01). 
Out of 64 suspected cases of tuberculosis, 8 cases were positive for AFB smear/ culture. Samples negative for both AFB smear/ culture were 56.

From these 8 samples, 5 samples were positive smear positive. Seven isolates of mycobacteria were obtained. A total of 4 samples were both smear and culture positive, while 3 samples were smear negative and culture positive. Only one case of a sputum sample was smear positive and culture negative.

All smear negative culture positive samples were pleural fluids. Smear positive, culture positive samples included 3 sputum and 1 BAL samples.

All the 7 isolates obtained were slow growers taking an average time of $32.88 \pm 12.07$ days for growth. All the isolates were non-chromogens. On the basis of the biochemical identification, all the isolates were identified as Mycobacterium tuberculosis.

The mean detection time taken for the isolation of mycobacteria on both the sets of LJ media was determined. The contamination rates on both these sets of media were also evaluated.

The contamination rate of LJ media with Petroff's method was $20.31 \%$, while the contamination rate with NALC-NaOH method was $14.06 \%$. A total of 8 sputum samples were contaminated out of 36 with Petroff's method, while 6 sputum samples were contaminated with NALC-NaOH method.

All the contaminants were detected within first week of incubation. Twenty-one LJ slants showed contamination (16 bacterial and 5 fungal). Majority of the bacterial contaminants were Pseudomonas species, Acinetobacter species, Escherichia coli and Staphylococcus aureus. Among the fungal contaminants, Aspergillus species, Mucor species and Candida species were common.

As far as bronchoalveolar lavage was concerned 2 out of 6 samples were contaminated with Petroff's method, while 1 was contaminated with NALC-NaOH method.

Out of the total 10 urine samples processed 3 samples were contaminated with Petroff's method, while only 2 were contaminated with NALC-NaOH method.

With Petroff's method 8 out of the total 36 sputum samples were contaminated, while with NALC-NaOH method only 6 samples were contaminated.

In our study, the rate of isolation was similar in both the decontamination procedures. The rate of isolation was $10.93 \%$ on both the sets of LJ media. The sensitivity of smear was $57.14 \%$ and sensitivity of culture was $80 \%$. The specificity of smear was $98.24 \%$.

\begin{tabular}{|c|c|}
\hline Total Samples & $\mathbf{6 4}$ \\
\hline Sputum & 36 \\
\hline BAL & 06 \\
\hline Pleural Fluid & 07 \\
\hline Urine & 10 \\
\hline Endometrial Biopsy/ Curettage & 2 \\
\hline Bone Marrow Biopsy & 1 \\
\hline ET & 1 \\
\hline Nasal Secretion & 1 \\
\hline \multicolumn{2}{|c|}{ Table 1. Sample-Wise Distribution } \\
\hline
\end{tabular}

\begin{tabular}{|c|c|c|}
\hline Sample & $\begin{array}{c}\text { Petroff's } \\
\text { Method }\end{array}$ & $\begin{array}{c}\text { NALC-NaOH } \\
\text { Method }\end{array}$ \\
\hline Sputum & 8 & 6 \\
\hline BAL & 2 & 1 \\
\hline Pleural Fluid & - & - \\
\hline Urine & 3 & 2 \\
\hline $\begin{array}{c}\text { Endometrial Biopsy/ } \\
\text { Curettage }\end{array}$ & - & - \\
\hline Bone Marrow Biopsy & - & - \\
\hline ET & - & - \\
\hline Nasal Secretion & - & - \\
\hline Contamination Rate & $\mathbf{2 0 . 3 1 \%}$ & $\mathbf{1 4 . 0 6 \%}$ \\
\hline \multicolumn{2}{|c|}{ Table 2. Contamination on LI Medium } \\
\hline
\end{tabular}

\begin{tabular}{|c|c|c|}
\hline & Culture Positive & Culture Negative \\
\hline Smear Negative & $\begin{array}{c}\text { 3 (All were pleural } \\
\text { fluids) }\end{array}$ & 56 \\
\hline Smear Positive & 4 (3-Sputum; 1-BAL) & 1 (Sputum) \\
\hline Total & $\mathbf{7}$ & $\mathbf{5 7}$ \\
\hline \multicolumn{3}{|c|}{ Table 3. Isolation Rate } \\
\hline
\end{tabular}

\section{DISCUSSION}

TB was described by Hippocrates (400 B.C.) in "Of the Epidemics" and was clearly documented by Claudius Galen during the Roman Empire. "Consumption, King's Evil, lupus vulgaris and phthisis" are some of the names for TB that have been used in the last several centuries.[8] With the introduction of the first tuberculosis agents, streptomycin in 1944, p-aminosalicylic acid (PAS) in 1946 and INH in 1952, control of tuberculosis seemed a possibility. However, despite these drugs and the subsequent introduction of many additional effective antitubercular agents, eradication of tuberculosis has remained elusive.[9]

Tuberculosis, as yet is far from being controlled.[10] The diagnosis of mycobacterial infections remained practically unchanged for many decades and probably would have not progressed at all without the unexpected resurgence of $\mathrm{TB}$, which characterised the last twenty years of the 20th century.[11]

The present trend and pattern of MTB infection which include its opportunistic and lethal synergy with HIV, therapeutic complications due to emergence of drug resistant MTB strains and infection by non-tuberculous mycobacteria (NTM) species necessitate the scaling up of routine diagnostic procedures at least to include culture and identification of causative mycobacterial species.

The classic laboratory approach to the diagnosis of mycobacterial infections involves the phenotypic characterisation of colonies growing on LJ medium. ${ }^{[4]}$ Examination by mycobacterial culture provides the only definitive diagnosis of tuberculosis.[3]

Isolation of Mycobacteria from specimens contaminated with normal flora like sputum poses a problem in view of high nutritive value of the egg media used for mycobacterial culture. Specimens are treated to kill various non-acid fast organisms by decontamination techniques. Different laboratories use different decontamination techniques. No single technique is entirely satisfactory. Hence, this study was conducted to compare the Petroff's method and NALC-NAOH (N-Acetyl-L-cysteine $\mathrm{NaOH}$ ) method digestion decontamination procedures. 
Dorothy et al had compared the contamination rate with four different decontamination methods. They found that contamination rate was $24 \%$ and $25 \%$ with Petroff's and NALC-NaOH methods respectively.[12] Present study showed that NALC $(14.06 \%)$ is a better decontamination method in comparison to Petroff's (20.31\%). The difference in the contamination rate was found to be statistically significant. Pearson Chi-square test was applied. Its value was 32.267 and $\mathrm{p}=0.000$.

Damle and DV had shown that in Petroff's method, the rate of isolation was $80.7 \%$ (168 strains), while in NALC$\mathrm{NaOH}$ method the yield was $78.8 \%$. However, in the present study no such difference was found. In our study, the rate of isolation was $10.93 \%$ on both the sets of LJ media.[13]

The sensitivity of smear was $80 \%$, while the specificity of smear was $94.9 \%$. This is in agreement with other studies. According to another study, the sensitivity of smear microscopy is low $(40 \%-60 \%)$ and particularly low for diagnosing tuberculosis associated with HIV infection and disease in children.[14] Zia et al have reported a sensitivity of $51 \%$ for the ZN staining.[15] According to Ulukanligil et al, sensitivity of ZN staining was $67 \%$. $^{[16]}$

A study conducted in Iran had reported the sensitivity and specificity of $51 \%$ and $100 \%$ for the $\mathrm{ZN}$ staining method.[15] A study conducted by Ulukanligil $\mathrm{M}$ et al reported a sensitivity of $61 \%$ with ZN staining and specificity of $100 \%$.[16] This is in concordance with our study. We reported the sensitivity of ZN staining to be $57.14 \%$. The specificity of ZN staining was $99.64 \%$.

As per our study, the sensitivity of culture on LJ medium was $57.14 \%$. However, in literature sensitivity of LJ medium had been reported to be $59.7 \%, 76.9 \%$ and $87 \% .[17,18,19]$

The mean detection time for isolation of mycobacteria on LJ media was $32.88 \pm 12.07$ days. It was then separately computed for AFB smear negative and AFB smear positive specimens.

The mean detection time was $39.71 \pm 2.73$ days for growth of AFB smear negative samples, while it was $30.22 \pm$ 12.76 days for AFB smear positive specimens which was found to be statistically significant ( $p=0.0070)$.

A Malaysian study had shown the mean detection time to be 33 days with LJ medium.[20] Chew et al had shown the mean detection time to be 27 days with LJ medium.[18] Our findings were in agreement to other studies.

The mean detection time of growth on LJ media for AFB smear positive samples was 30.22 days as compared to 39.71 days for AFB smear negative samples. This was similar to a study, which reported the values to be 31.2 days and 35.3 days respectively.[20]

The core problem is that existing microscopy methods to diagnose TB are both technically and practically inadequate for use in high-burden countries. Microscopy is too complex to implement and too slow to perform to be used as a pointof-care test. The primary clinics where most patients with symptoms of TB first seek care rarely have AFB microscopy available and patients have to wait until they are referred to a specialty clinic or microscopy centre. Even then most TB cases will be missed, both because of the inherent limits of the sensitivity of the test which detects only $60 \%$ of culturepositive pulmonary TB patients even in clinical trial settings and because of the logistical difficulties of making quality assured microscopy widely and freely available. Moreover, microscopy misses exactly the patients those with early disease that one would like to detect and put on treatment to block transmission before it has begun.[21]

The clinical microbiology laboratory has a critical role in the detection and control of infection caused by clinically significant Mycobacterium species. Rapid, sensitive and accurate detection of these organisms in clinical specimens can hasten the administration of appropriate antimycobacterial therapy and prevent the spread of infection to susceptible contacts through the use of infection control practices.

Conventional solid media such as Lowenstein-Jensen media traditionally have been used for the recovery of mycobacteria from clinical specimens. However, the slow rate of growth of many pathogenic Mycobacterium species on solid media can substantially delay the identification process. Broth media such as Middlebrook 7H9 have been developed to speed the growth and recovery rate of mycobacteria in the laboratory.

However, there is an increase in costs compared to those of conventional cultivation techniques. This must be weighed against the benefits gained by early diagnosis and the higher sensitivity of detection. Tuberculosis, one of the oldest recorded human afflictions is still one of the biggest killers among the infectious diseases, despite the worldwide use of a live attenuated vaccine and several antibiotics.[22] Tuberculosis, as yet is far from being controlled. Several reasons can be attributed to this, a major contributing factor being the development of resistance to the currently available drugs due to the successful adaptation of the pathogen.

Hence, to conclude tuberculosis still poses a diagnostic challenge both for the Microbiologists and the clinicians. There is a constant need to formulate newer methods of diagnosis and to improve upon the existing methods. This can be achieved by more exhaustive and thorough research.

\section{CONCLUSION}

We observed that there was no significant difference in the isolation rate of mycobacteria with the use of either Petroff's method or NALC-NaOH method, while the contamination rate was higher with Petroff's method.

\section{REFERENCES}

[1] TB India 2010: RNTCP status report. Available from: http://www.tbcindia.org 2010.

[2] Revised national tuberculosis control programme: DOTS plus guidelines 2008. Available from: http://www.tbcindia.org 2010.

[3] Laboratory services in tuberculosis control organization and management part I. Available from: WHO/TB/98.25. 2010.

[4] Winn JW, Allen S, Janda W, et al. Mycobacteria. In: Koneman's color atlas \& textbook of diagnostic microbiology. $6^{\text {th }}$ edn. Lippincot Williams \& Wilkins. United States of America 2006:1065-117.

[5] Revised national TB control programme manual for sputum smear fluorescence microscopy 1-16.

[6] Forbes BA, Sahm DF, Weissfeld AS. Mycobacteria. In: Bailey \& Scott's diagnostic mirobiology. $12^{\text {th }}$ edn. Mosby Elsevier. China 2007:478-508. 
[7] Walt B, Rayner A, Harris G. Mycobacterium. In: Mackie \& McCartney practical medical microbiology. Collee JG, Fraser AG, Marmion BP, et al. (eds). 14th edn. Churchill Livingstone. India 2007:329-40.

[8] Mathema B, Kurepina NE, Bifani PJ, et al. Molecular epidemiology of tuberculosis: current insights. Clin Microbiol Rev 2006;19(4):658-85.

[9] Sepkowitz KA, Raffalli J, Riley L, et al. Tuberculosis in the AIDS era. Clin Microbiol Rev 1995;8(2):180-99.

[10] Manjula S, Sritharan V. Microbial pathogenesis: an insight into mycobacterium tuberculosis. Ind J Med Microbiol 2002;20(2):61-8.

[11] Tortoli E, Palomino JC. New diagnostic methods. In: Tuberculosis. Palomino JC, Leao SC, Ritacco V. (eds). $1^{\text {st }}$ edn. 2007:441-86.

[12] Yeboah-Manu D, Bodmer T, Mensah-Quainoo E, et al. Evaluation of decontamination methods and growth media for primary isolation of mycobacterium ulcerans from surgical specimens. J Clin Microbiol 2004;42(12):5875-6.

[13] Damle AS, Kaundinya DV. Comparison of three methods of decontamination of sputum for mycobacterial culture. Ind J Tub 1986;33(3):125-8.

[14] Stop TB new diagnostics working group strategic plan 2006-2015. Available from: http://www.stoptb.org/wg/new_diagnostics/ assets/ documents/SP\%20Stop\%20TB\%20Dia\%20WG\%20FINAL-Dec2005.pdf. 2010.

[15] Ziaee M, Namaei M, Khazaei M, et al. Comparison of the value of two different sputum staining for diagnosis of acid-fast bacilli. Iranian J Clin Infect Dis 2008;3(2):99-102.
[16] Ulukanligil M, Aslan G, Tasçi S. A comparative study on the different staining methods and number of specimens for the detection of acid fast bacilli. Mem Inst Oswaldo Cruz 2000;95(6):855-8.

[17] Lu D, Heeren B, Dunne WM. Comparison of the automated mycobacteria growth indicator tube system (BACTEC 960/MGIT) with lowenstein-jensen medium for recovery of mycobacteria from clinical specimens. Am J Clin Pathol 2002;118(4):542-5.

[18] Chew WK, Lasaitis RM, Schio FA, et al. Clinical evaluation of the mycobacteria growth indicator tube (MGIT) compared with radiometric (BACTEC) and solid media for isolation of mycobacterium species. J Med Microbiol 1998;47(9):821-7.

[19] Badak FZ, Kiska DL, Setterquist S, et al. Comparison of mycobacteria growth indicator tube with BACTEC 460 for detection and recovery of mycobacteria from clinical specimens. J Clin Microbiol 1996;34(9):22369.

[20] Nor FM, Peng NGK, Fong Y. The manual MGIT system for the detection of $M$. tuberculosis in respiratory specimens: an experience in the university Malaya medical centre. Malaysian J Pathol 2009;31(2):93-7.

[21] Young DB, Perkins MD, Duncan K, et al. Confronting the scientific obstacles to global control of tuberculosis. J Clin Invest 2008;118(4):1255-65.

[22] Smith I. Mycobacterium tuberculosis pathogenesis and molecular determinants of virulence. Clin Microbiol Rev 2003;16(3):463-96. 